\title{
Prospects of terahertz technology in diagnosis of human brain tumors - A review
}

\author{
Guzel R. Musina ${ }^{1}$, Pavel V. Nikitin ${ }^{2}$, Nikita V. Chernomyrdinn', Irina N. Dolganova ${ }^{3,4}$, \\ Arsenii A. Gavdush ${ }^{1}$, Gennadiy A. Komandin ${ }^{1}$, Dmitry S. Ponomarev5 , Alexander A. Potapov², \\ Igor V. Reshetov ${ }^{6}$, Valery V. Tuchin ${ }^{7,8}$, and Kirill I. Zaytsev ${ }^{1,9 *}$ \\ ${ }^{1}$ Prokhorov General Physics Institute of the Russian Academy of Sciences, 38 Vavilova str., Moscow 119991, Russia \\ ${ }^{2}$ Burdenko Neurosurgery Institute, 14 -ya Tverskaya-Yamskaya str., Moscow 125047, Russia \\ ${ }^{3}$ Institute of Solid State Physics of the Russian Academy of Sciences, 2 Academika Osipyana str., Chernogolovka, \\ Moscow Region 142432, Russia \\ ${ }^{4}$ Institute for Regenerative Medicine, Sechenov University, 19/1 Bolshaya Pirogovskaya str., Moscow 119146, Russia \\ ${ }^{5}$ V.G. Mokerov Institute of Ultra High Frequency Semiconductor Electronics of the Russian Academy of Sciences, \\ 7 Nagorniy proezd, Moscow 117105, Russia \\ ${ }^{6}$ Institute for Cluster Oncology, Sechenov University, 19/1 Bolshaya Pirogovskaya str., Moscow 119146, Russia \\ ${ }^{7}$ Saratov State University, 83 Astrakhanskaya str., Saratov 410012, Russia \\ ${ }^{8}$ Institute of Precision Mechanics and Control of the Russian Academy of Sciences, 24 Rabochaua str., Saratov 410028, \\ Russia \\ ${ }^{9}$ Bauman Moscow State Technical University, 5/1 2-ya Baumanskaya str., Moscow 105005, Russia \\ *e-mail: kirzay@gmail.com
}

\begin{abstract}
Terahertz (THz) waves feature high sensitivity to the content and state of water in biological tissues. Therefore, during the past decades, $\mathrm{THz}$ technology has attracted significant attention in biophotonics, including diagnosis of malignant and benign neoplasms with different nosologies and localizations. The pathophysiological features of malignant tumors of the central nervous system determine appearance of several morphological phenomena, such as increased vascularity, edema, necrosis. These phenomena cause water content increase in the studied tissues and, thus, open new ways for the $\mathrm{THz}$ technology applications in the intraoperative neurodiagnosis, including delineation of tumor margins. This research area is rather novel and, despite the small amount of accumulated research material, is undoubtedly extremely promising for creation of new diagnostic approaches. In this review, available results in the considered exciting branch of $\mathrm{THz}$ technology are summarized, and potential projections of this topic into the future are constructed. (C) 2020 Journal of Biomedical Photonics \& Engineering.
\end{abstract}

Keywords: terahertz radiation; terahertz Biophotonics; terahertz spectroscopy; terahertz imaging; brain tumors; glioma; meningioma; neurodiagnostics; neurosurgery.

Paper \#3375 received 3 Jun 2020; accepted for publication 18 Jun 2020; published online 28 Jun 2020. doi: 10.18287/JBPE20.06.020201.

\section{Introduction}

Malignant gliomas, which represent the largest proportion of malignant tumors of the central nervous system (CNS), are an important problem in modern oncology [1]. Neurosurgical intervention remains the most effective method of glioma treatment, while patient prognosis is largely determined by the grosstotal resection of a tumor [2]. At the same time, there are several factors limiting the possibilities of radical 
resection, among which we would mention the two most important ones [3]:

- high risk of adverse functional outcomes with severe neurological deficiency,

- a problem of determining the true margins of a tumor due to its infiltrative growth.

If the first problem can be mitigated using electrophysiological methods and functional magnetic resonance imaging, the second one is left to the urgent histological examination, which is characterized by relatively low information content and a high percentage of errors [4]. Therefore, development of fundamentally novel intraoperative diagnostic approaches to identify the tumor margins remains an important one.

Few experimental methods have been introduced recently based on the different physical and metabolic characteristics of the tumor tissues, in order to determine the true margins of a tumor. In particular, the metabolic aspect is based on the use of 5-AminoLevulinic Acid (5-ALA)-induced fluorescence of Protoporphyrin IX, which is most actively accumulated in the tumor tissue due to a higher level of metabolism, allowing us for assessing the brain tumor margins. However, the sensitivity and specificity of this method is significantly reduced due to aberrant accumulation of 5-ALA in non-tumor tissues with inflammation, increased vascular permeability, edema, and other causes, as well as to its poor accumulation in low-grade gliomas [5]. At the same time, other methods are vigorously explored nowadays. There are Raman spectroscopy and imaging [6], photoacoustic imaging [7], multiphoton microscopy and optical coherence tomography (OCT) [8,9]. Nevertheless, application of these techniques in neurosurgical practice is still difficult, so they remain instruments of basic research. Therefore, further development of new tools for the intraoperative delineation of tumor margins is a challenging problem.

Thanks to a high sensitivity of the terahertz (THz) waves to the content and state of water in tissues, $\mathrm{THz}$ spectroscopy and imaging can be applied for the labelfree differentiation between healthy tissues and neoplasms with different nosologies and localizations. Among all applications of $\mathrm{THz}$ technology in oncodiagnosis, during the last few years, it attracted attention as a tool for the intraoperative delineation of brain tumor margins. Despite quite a small amount of research material accumulated in this exciting branch of $\mathrm{THz}$ technology, it is undoubtedly extremely promising for creation of new diagnostic approaches. In this review, modern research results in the area of intraoperative $\mathrm{THz}$ diagnosis of brain tumors are discussed.

\section{Terahertz biophotonics}

Progress in femtosecond laser technologies and creation of new methods for the THz-wave generation and detection have been contributed to the recent development of THz spectroscopy and imaging [10]. As shown in Fig. 1, THz radiation belongs to the frequency range of $0.1-3.0 \mathrm{THz}$ or to the wavelength range of $3 \mathrm{~mm}-100 \mu \mathrm{m}$. Since the end of the XX century, THz spectroscopy and imaging have been the subject of research to create a fundamentally new approach to noninvasive and invasive oncodiagnostics. This approach has been successfully implemented on neoplasms of the skin [11], mucous [12], colon [13], breast [14], stomach [15], liver [16], etc. Most recently, high potential of $\mathrm{THz}$ technology was demonstrated in differentiation of intact (healthy) tissue and tumors of the brain [17].

Let us start with fundamental aspects of the THzradiation - biological tissues interactions. As shown in Fig. 2, structural elements of tissues (such as microfibrils, separate cells and their agglomerates, cell organelles, etc.) are too small at the scale posed by $\mathrm{THz}$ wavelengths. Conventional $\mathrm{THz}$ imaging systems, which mostly rely on the diffraction limited lens- or mirror-based optics, provide the spatial resolution up to $\sim \lambda$ and, thus, do not allow to resolve such small scale tissue components. As a result, only the effective response of tissues at $\mathrm{THz}$ frequencies, which is averaged within $>\lambda^{2}$ area of the THz beam spot, can be studied using $\mathrm{THz}$ spectroscopy and imaging [18]. In this way, the electrodynamic properties of tissues in the $\mathrm{THz}$ range are usually described using the frequencydependent effective complex dielectric permittivity

$$
\tilde{\varepsilon}=\varepsilon^{\prime}-i \varepsilon^{\prime \prime}
$$

which includes both real $\varepsilon^{\prime}$ and $\varepsilon^{\prime \prime}$ imaginary parts. At the same time, it can be represented by a frequencydependent complex refractive index

$$
\tilde{n}=n^{\prime}-i n^{\prime \prime} \equiv n-i \frac{c}{2 \pi v} \alpha \equiv \sqrt{\tilde{\varepsilon}}
$$

where $n \equiv n^{\prime}$ and $n^{\prime \prime}$ are real and imaginary parts, $c=3 \times 10^{8} \mathrm{~m} / \mathrm{s}$ is the speed of light in a free space, $v$ is an electromagnetic wave frequency in $[\mathrm{Hz}]$, and $\alpha$ is absorption coefficient (by field) in $\left[\mathrm{cm}^{-1}\right]$.

Biological liquids and tissues, water and its solutions do not feature resonant spectral absorption peaks in the $\mathrm{THz}$ range. The main factor determining the $\mathrm{THz}$ dielectric response of healthy and pathological tissues (either in vivo or freshly-excised (non-dehydrated) specimens ex vivo) is the content of free and bound water in tissues [18]. The THz-wave-tissue interactions can be completely described in the frameworks of electrodynamics using the relaxation models of complex dielectric permittivity (such as the Debye, Gavrilyak-Negami, Cole-Cole, Davidson-Cole models [19-24]), while the double-Debye model remains the most frequently-applied one in $\mathrm{THz}$ biophotonics $[18,23,24]$

$$
\tilde{\varepsilon}=\varepsilon_{\infty}+\frac{\Delta \varepsilon_{1}}{1+i 2 \pi v \tau_{1}}+\frac{\Delta \varepsilon_{2}}{1+i 2 \pi v \tau_{2}}
$$




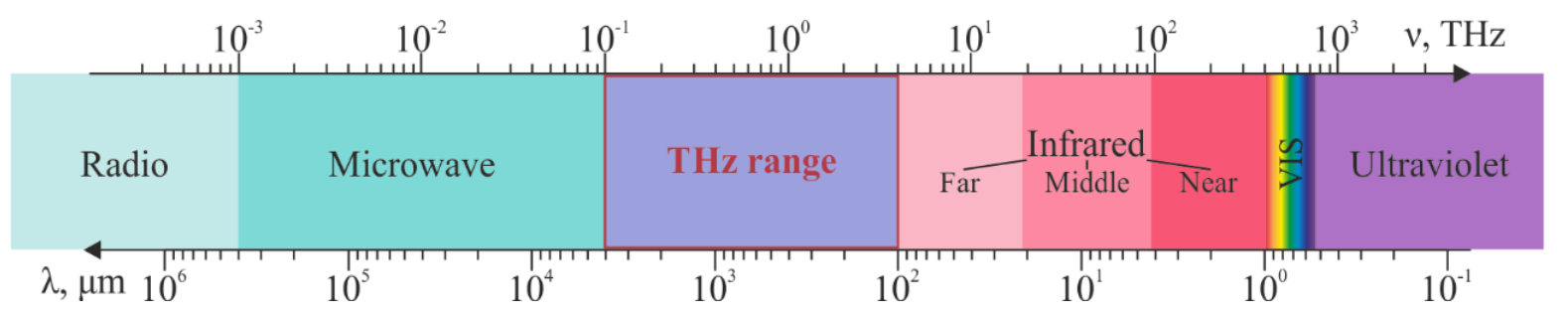

Fig. $1 \mathrm{THz}$ range of the electromagnetic spectrum. Courtesy of G.R. Musina.

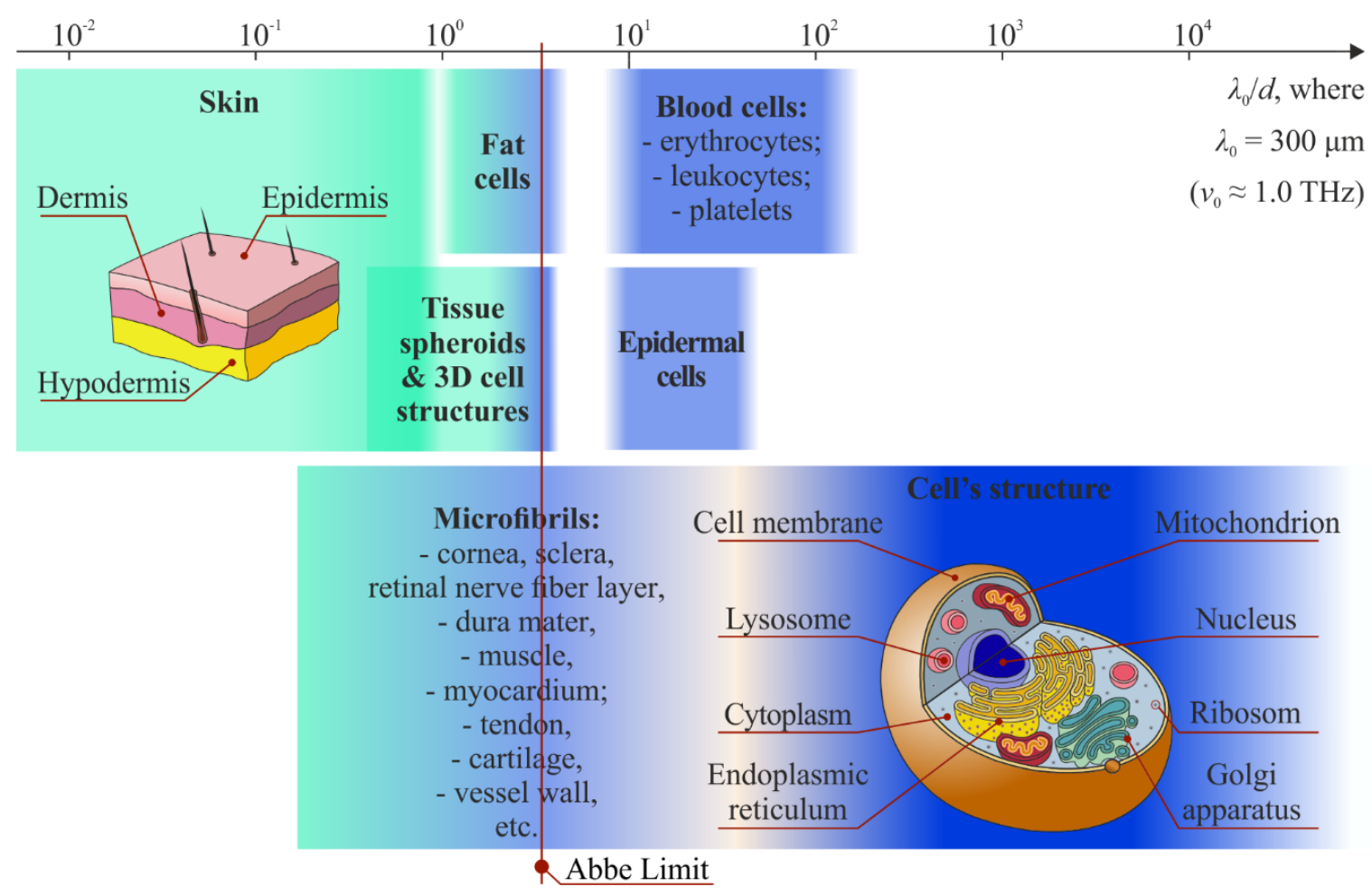

Fig. 2 Dimensions of tissue structural elements $\delta$ as compared to the THz wavelength of $\lambda_{0}=300 \mu \mathrm{m}$ (it corresponds to the frequency of $v_{0}=1.0 \mathrm{THz}$ ). By vertical red line, the $\lambda / 2$ Abbe diffraction limit is shown. Courtesy of K.I. Zaytsev.

here, $\varepsilon_{\infty}$ is a dielectric permittivity at infinitely-high frequencies as compared to the considered frequency band); $\tau_{1}$ and $\tau_{2}$ are relaxation times (in [ps]) corresponding to the "slow" and "fast" processes; $\Delta \varepsilon_{1}$ and $\Delta \varepsilon_{2}$ regulate contributions of "slow" and "fast" Debye relaxation terms to the complex dielectric response function [18]. This double-Debye model allows for convenient parameterization of the tissue dielectric properties in the entire $\mathrm{THz}$ range by using only 5 independent constants: $\varepsilon_{\infty}, \Delta \varepsilon_{1}, \Delta \varepsilon_{2}, \tau_{1}$, and $\tau_{2}$. However, this model is not physically rigorous, since it does not fulfill the summation rule, and it approximates the experimental data by the two broad absorption bands, the maxima of which are centered at the frequencies $\sim \tau_{1}^{-1}, \sim \tau_{2}^{-1}$ located outside (or at the edges) of the analyzed spectral range. Thus, the considered double-Debye model implies extrapolation of the experimental data. However, this model provides quite an effective parameterization tool, and it also can be applied for physically reasonable differentiation of tissues [23-27].

Majority of biological tissues possesses high content of water due to several histogenetic reasons. For example, fibrous tissues contain significantly large amount of water, as compared to that in adipose tissues. High water content strongly affects the $\mathrm{THz}$ dielectric properties of tissues. Since water molecule is very polar, both liquid and tissue water strongly absorbs $\mathrm{THz}$ waves, thus, reducing the depth of THz-wave penetration in tissues. The depth of tissues probing by $\mathrm{THz}$ radiation can be as large as tens or hundreds of microns, depending on the considered frequency and the tissue type. Such a small penetration depth forms a key limitation of $\mathrm{THz}$ technology use for studying biological tissues in various branches of biophotonics. In order to obtain reliable results, response of tissue at $\mathrm{THz}$ frequencies is usually studied in reflection geometry [28].

There are several factors that determine the ability to differentiate intact and tumor tissue in the $\mathrm{THz}$ range. The key one is the different water content. Due to higher level of metabolism, edema and increased vascular permeability, tumor tissues contain more water than normal ones, which leads to higher refractive index 
and absorption coefficient of a tumor in the $\mathrm{THz}$ range [17]. At the same time, there are other factors that impact the $\mathrm{THz}$ response of tissues tissue and that can be of considerable practical interest. In particular, the presence of necrotic debris, which is a morphological sign for many malignant tumors (for example, glioblastoma), is among such factors [29]. Furthermore, additional changes in the $\mathrm{THz}$ dielectric response of tissues can be attributed to some mutational changes, the systematic study of which is of great practical interest [30].

The complex dielectric permittivity $\tilde{\varepsilon}$ (see Eq. (1)) or the complex refractive index $\tilde{n}$ (see Eq. (2)) completely describe the THz-radiation - biological tissues interactions in the framework of classical electrodynamics. Such a complete description allows us to:

- model analytically the THz-wave propagation through multi-layer media, including biological tissues, using the plane wave approximation, the Fresnel formulas, and the modified Bouguer-Lambert-Beer law, which is of crucial importance for $\mathrm{THz}$ spectroscopy [17];

- simulate numerically the THz-wave interaction with biological objects, featuring complex geometry and heterogenous character, using computational method of electrodynamics [23] or statistical Monte Carlo techniques [31];

- apply the complex dielectric permittivity, related derived quantities or even parameter of relaxation dielectric permittivity models (see Eq. (3)) for the discrimination between different tissue types [17, 27].

It is worth noting that biological tissues can possess significant heterogeneity and complexity of the internal structure even at the scale posed by $\mathrm{THz}$ wavelength (see Fig. 2), which is not taken into account in the effective medium theory formalism and which could not be described by the effective dielectric response function (see Eq. (6)). As examples of such heterogenous tissues, one can consider results of $\mathrm{THz}$ microscopy of individual fat cells embedded into fibrous connective mammary gland ex vivo, as well as muscle fibers of the tongue ex vivo from Ref. [32]. For such structural inhomogeneities of tissues, one can expect Mie scattering of $\mathrm{THz}$ waves [33], in contrast to the Rayleigh scattering regime considered in the effective medium theory [34]. Dispersion, absorption and scattering properties of such tissues should be simultaneously taken into account in order to form novel approach for describing the THz-wave - tissue interactions [33], while the radiation transfer theory should be considered as a theoretical basis for this purpose [35]. Nevertheless, problems of studying the scattering properties of tissue and of developing the radiation transfer theory in the $\mathrm{THz}$ range remain unaddressed.

With a rapid progress in $\mathrm{THz}$ pulsed spectroscopy and imaging observed at the end of the XX century, $\mathrm{THz}$ technology are extensively applied in biology and medicine, but it is still far from clinical practice owing to several reasons $[18,36]$. In the next few sections, modern research results in the area of $\mathrm{THz}$ diagnostics of brain tumors are analyzed, and problems and difficulties that stand in the way of $\mathrm{THz}$ technology translation into a practical plane are discussed.

\section{Terahertz spectroscopy and imaging of brain tumors}

Let us start with one of the first research papers in $\mathrm{THz}$ neurodiagnosis [37]. Using $\mathrm{THz}$ pulsed spectroscopy in conjunction with a closed cycle cryostat, the authors of this work demonstrated an ability for differentiation between intact and pathologically altered frozen human brain tissue with amyloid plaques ex vivo, which are inherent to the Alzheimer's disease. Thanks to tissue freezing, THz-wave absorption by tissue water is strongly suppressed [17], which allows revealing nonwater-related differences in the $\mathrm{THz}$ dielectric properties of normal and pathologically altered tissues, such as the amyloid plaque proteins. Next, THz spectroscopy was applied for studying a mouse model of the Alzheimer's disease [38], and the observed data for dehydrated brain tissues ex vivo confirmed earlierreported results and indicated a significant effect of the Alzheimer's disease-related amyloid proteins on the $\mathrm{THz}$ dielectric response of tissues.

An ability of using $\mathrm{THz}$ technologies for the diagnosis of human brain tumors is attracted considerable attention most recently, starting from tumor models in mice and rats [29, 39].

\subsection{Glioma models in mice and rats}

The orthotopic rat glioma model was studied using the $\mathrm{THz}$ pulsed spectrometer equipped with a raster scan unit in Ref. [29], where 9 week old male rats received 9L/lacZ cells, and the tumor was grown for 3-4 weeks to a volume of $\sim 1.0 \mathrm{~cm}^{3}$ [40]. A distinctive feature of this model is a clear boundary between the tumor and intact brain tissues that simplifies their visualization and discrimination. After extraction, the rat brains were divided into equal parts. Then, $\mathrm{THz}$ images of tissue specimens were collected and formed at each point using peak values of the $\mathrm{THz}$ waveforms (see Fig. 3) [29]. The tumor margins revealed by such $\mathrm{THz}$ imaging are well comparable with that obtained by magneticresonance tomography and white light imaging. The results of this study have highlighted ability for the intraoperative diagnosis of brain tumors using $\mathrm{THz}$ technology.

At the same time, it seems to be important to study other factors, besides water content in tissues that underlie the contrast between tumor and intact brain tissues in the $\mathrm{THz}$ range. For this aim, it is necessary to eliminate water from biological tissues that was achieved in Ref. [29] by paraffin embedding of tissues. By further $\mathrm{THz}$ imaging of paraffin-embedded tissues and by comparing the obtained patterns with the results of microscopic examination of the same tissue specimens stained with Hematoxylin and Eosin (H\&E), 


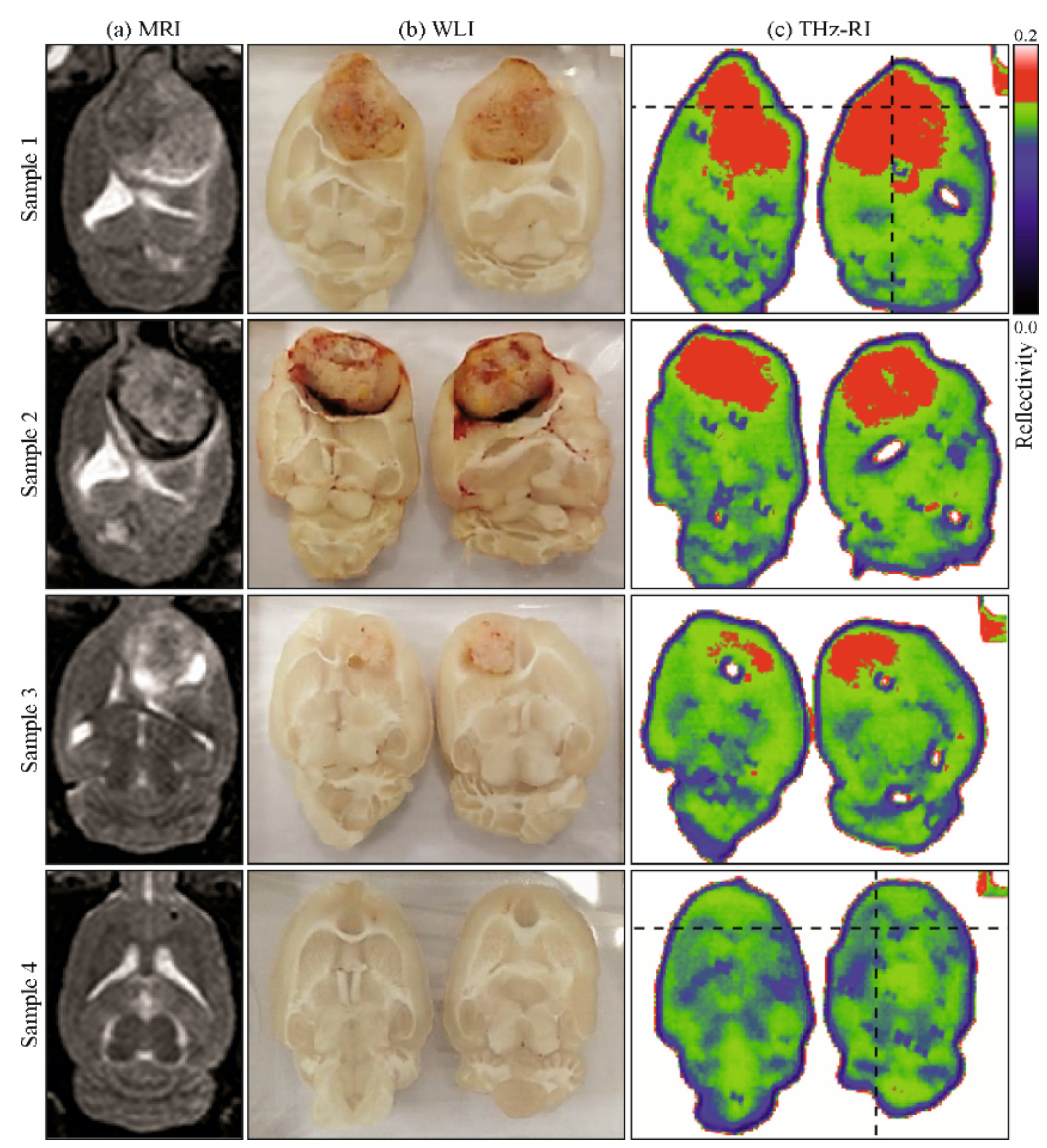

Fig. 3 (a) Magnetic resonance images (MRI), (b) white light images (WLI), and (c) THz reflection-mode images (THz-RI) of freshly-excised whole rat brain tissues, both with orthotopic glioma model (rows 1-3) and without a tumor (row 4). Adapted with permission from Ref. [29]. (C) The Optical Society of America.

it was found that a differences between intact and pathologically altered tissues are still presented in $\mathrm{THz}$ images, but this difference is several tines lower that that observed for the freshly-excised rat brain tissues ex vivo. In some cases, the determination of true tumor margins relying on $\mathrm{THz}$ images of paraffin-embedded tissues was impossible. It should be noticed that $\mathrm{THz}$ refractive index of paraffin-embedded samples was higher near the tumor margins, which can be attributed to a higher cell density in this area as compared to the neoplasm core. Thus, in addition to free water, other factors affect the response of tissues in the $\mathrm{THz}$ range. A possible influence of necrotic debris in tumor tissues on their THz response was also indicated in Ref. [29].

In Ref. [29], by using a rat brain model ex vivo, it was shown that $\mathrm{THz}$ dielectric properties of white matter and gray matter differs, which can be attributed to various content of myelin in these two types of brain tissues. Myelin forms a shell of axons, providing conditions for an adequate conduction of an impulse along them. Therefore, it is presented in a higher amount in white matter that leads to changes in its $\mathrm{THz}$ optical properties, as compared to that of a gray matter. This study has an important consequence in relation to pathological changes in brain tissue associated with changes in myelin content. THz technologies have good prospects for implementation in the diagnosis of multiple sclerosis. Nevertheless, the difference in the $\mathrm{THz}$ dielectric properties associated with myelin does not affect the possibility of detecting the tumor margins in both gray matter and white matter [41].

In Ref. [39], similar study was carried out using a glioma model in mice. For this aim, the GL261 glioma cell line was implanted into the brain of 6 week old mouse. The tumor was grown for 20 days, after which the brain was removed, cut into pieces and embedded into paraffin blocks. By studying these paraffinembedded tissues, the authors of Ref. [39] were able to clearly differentiate normal tissue and tumor using $\mathrm{THz}$ spectroscopy. In later studies, capabilities of $\mathrm{THz}$ technology in the intraoperative diagnostics of brain tumors was confirmed using $\mathrm{THz}$ pulsed spectroscopy and different types of gliomas. Particularly, freshly excised tissues of C6 glioma cell line ex vivo and human glioblastoma spheroids were studied in Refs. [42, 43]. At the same time, the observed results of $\mathrm{THz}$ spectroscopy and imaging of various glioma models require additional validation based on studies of the human brain gliomas.

\subsection{Human brain gliomas}

In order to mitigate this challenge, in Ref. [43], THz reflectometry was applied for studying ex vivo the human brain gliomas of the World Health Organization 


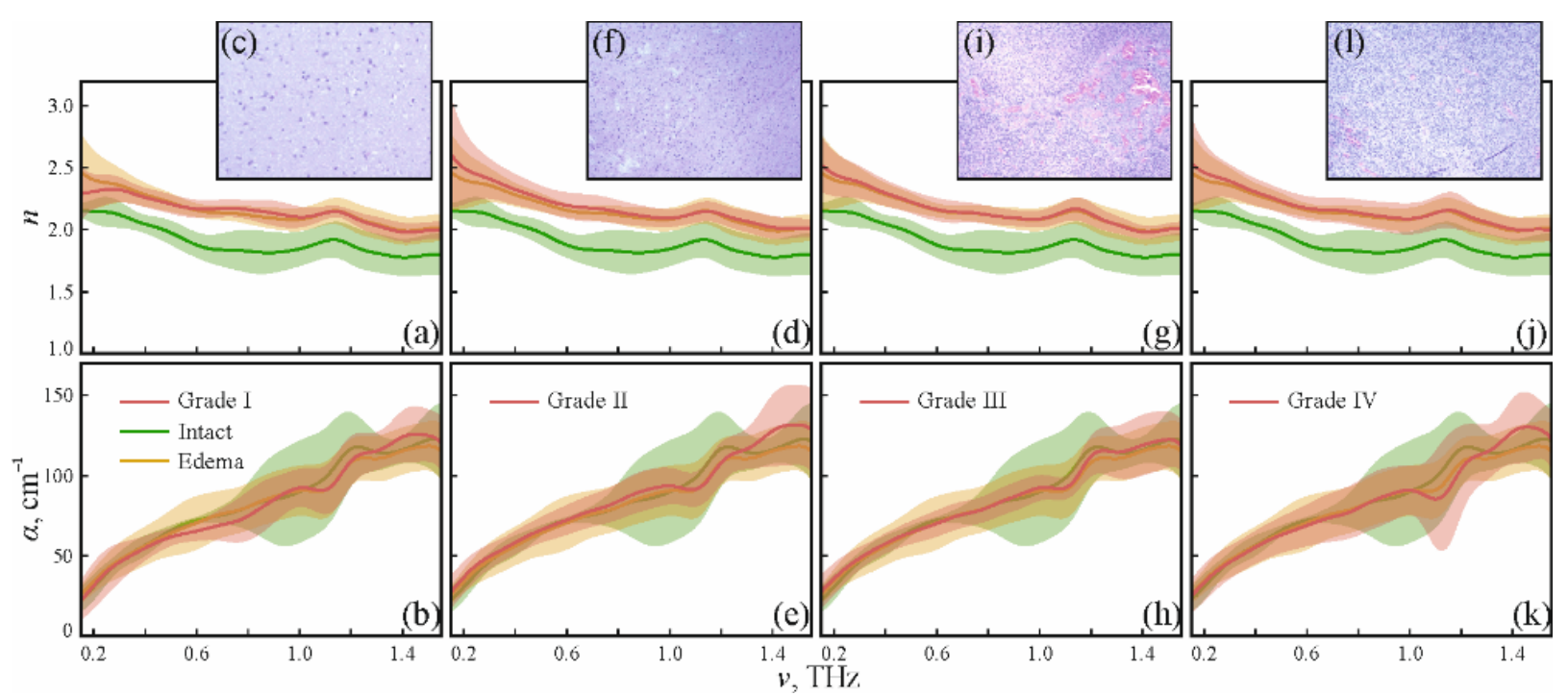

Fig. $4 \mathrm{THz}$ optical properties (i.e. refractive index $n$ and absorption coefficient $\alpha$ ) and H\&E-stained histology of gelatin-embedded human brain gliomas ex vivo of different WHO grade: (a)-(c) Grade I; (d)-(f) Grade II; (g)-(i) Grade III; (j)-(l) Grade IV. The error bars represent the 95\% confidential interval of measurements. Adapted from Ref. [17]), published by SPIE under a Creative Commons (CC BY) license.

(WHO) Grades II (6 samples), III (4 samples), and IV (4 samples) at $0.5 \mathrm{THz}$, thereby emphasizing the potential of $\mathrm{THz}$ reflection imaging for brain tumor diagnosis. At the same time, $\mathrm{THz}$ spectroscopy in a wide spectral range will open new prospects for the use of $\mathrm{THz}$ radiation for subsequent studies. It should be mentioned that $\mathrm{THz}$ spectroscopy and imaging may have a higher potential for determining the true tumor margins as compared to the 5-ALA-guided fluorescence spectroscopy and imaging. It was shown that in a cohort of patients with glioblastomas the tissue fluorescence was clearly observed only in half of the cases, while $\mathrm{THz}$ technology allowed us to determine the tumor margins in all the considered cases [44].

Next, in Ref. [45], intact tissues and human brain gliomas of WHO Grades I-IV were studied ex vivo using $\mathrm{THz}$ pulsed spectroscopy, while gelatin embedding of tissues was applied in order to prevent their hydration/dehydration and, thus, to sustain their $\mathrm{THz}$ dielectric response unaltered (as compared to that of freshly excised tissues ex vivo) over the entire period of tissue transportation and $\mathrm{THz}$ measurements. Moreover, fragments of perifocal brain tissue, including those in the edematous state, were also found in the investigated samples. After $\mathrm{THz}$ measurements, tissue fragments were fixed in formalin and sent to a routine histopathological study using the H\&E stained histology [17]. To study the $\mathrm{THz}$ response of human brain tissue, a reflection-mode $\mathrm{THz}$ pulsed spectrometer was used, the $\mathrm{THz}$ beam path of which was purged by nitrogen gas in order to suppress an impact of water vapors on the measured $\mathrm{THz}$ data. Details of brain tissue characterization can be found in Ref. [17].

In Fig. 4, the measured THz refractive index $n$ and absorption coefficient $\alpha$ are shown for the intact and edematous tissues, as well as human brain gliomas of different WHO Grades, while the insets show representative examples of tissue histology [17]. The error bars showed in Fig.4 accounts for fluctuations of the $\mathrm{THz}$ optical properties of tissue both within each tissue sample and within each tissue class, representing the $95 \%$ confidential interval of measurements. The statistical difference between the $\mathrm{THz}$ response of intact tissues and brain gliomas [17] (see Fig. 4) is fully consistent with the results of preliminary studies using glioma models from rats and mice [17, 39, 42, 43] (for example, see Fig. 3). A label-free contrast between the $\mathrm{THz}$ refractive index of intact tissues and tumors is observed [17, 43], while the refractive index is higher for a tumor. At the same time, distinguishing between edematous tissues and tumors, as well as between different WHO Grades of a tumor using $\mathrm{THz}$ spectroscopy, appears to be a daunting task. From Fig. 4, significant variations of the $\mathrm{THz}$ optical properties within each tissue class are clearly observed, which is usual for most modalities of the label-free tissue spectroscopy and imaging. A possible reason for such a high dispersion in the $\mathrm{THz}$ response of intact tissues can be due to the lack of differentiation between white matter and gray matter in the considered study (they form a single class of intact tissues in Fig. 4).

The results of Ref. [17] confirmed a strong potential of $\mathrm{THz}$ technology in the intraoperative diagnosis of human brain gliomas, where both $\mathrm{THz}$ spectroscopy and imaging can be applied for the label free detection of the tumor margins during surgery in order to ensure its gross total resection.

\subsection{Human brain meningiomas}

Other brain tumors were not spared. For example, in Ref. [44], it was shown that $\mathrm{THz}$ spectroscopy yields differentiation of intact brain tissues and meningiomas, as illustrated in Fig. 5. Nevertheless, the need of bringing $\mathrm{THz}$ technology to a clinical practice for 

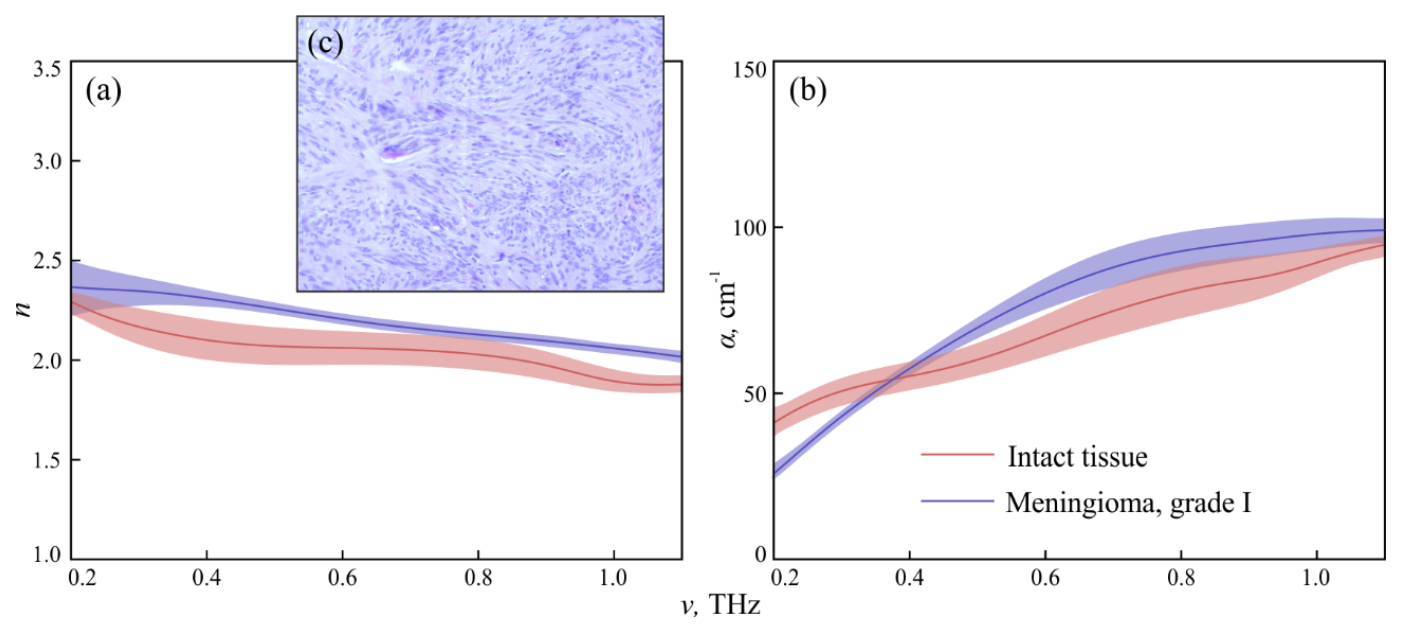

Fig. 5 Results of THz pulsed spectroscopy of gelatin-embedded human brain meningioma ex vivo of WHO Grade I: (a) refractive index $n$; (b) absorption coefficient $\alpha$, (c) H\&E-stained histology. Courtesy of K.I. Zaytsev.

diagnosis of other types of brain tumors (besides gliomas) remains a debatable issue, considering high costs of $\mathrm{THz}$ instruments.

\subsection{Determination of molecular characteristics of tumors}

$\mathrm{THz}$ technology can focus not only on the content and state of tissue water, but also on other molecular components, for example, myelin. There are other features of the molecular spectrum, which are reflected in the dielectric properties of tissues in the THz range. THz spectroscopy reveals the nucleotide composition of DNA and RNA. Changes in the composition of nucleotides and conformational changes in the DNA molecule generate low-frequency molecular vibrations that can be determined by $\mathrm{THz}$ spectroscopy. In principle, it is possible to determine the qualitative and quantitative composition of nucleotides using $\mathrm{THz}$ spectroscopy, since each of the four nucleotides has different absorption coefficients in the $\mathrm{THz}$ range [46, 47]. $\mathrm{THz}$ spectroscopy was used for the qualitative and quantitative analysis of various types of nucleic acids. It was shown that when the qualitative composition of the DNA chain is changed by one nucleotide, the spectral properties of the DNA molecule is changed in the $\mathrm{THz}$ range that indicates the possibility of detecting DNA point mutations using $\mathrm{THz}$ spectroscopy [48].

This potential of $\mathrm{THz}$ technology may be extremely useful for intraoperative diagnosis of brain tumors. There are a number of mutational changes, including point mutations, which are not only specific for certain types of CNS tumors, but also important factors that determine the patients prognosis. For example, point mutations of the IDH1 and $I D H 2$ genes are specific among CNS tumors for some diffuse gliomas: diffuse astrocytoma (Grade II), anaplastic astrocytoma (Grade III) and a special kind of glioblastoma (Grade IV) [49]. Moreover, the presence of this mutation determines a more favorable prognosis of the survival for patients with diffuse gliomas [50]. Another example is the presence of the BRAF V600E point mutation, which is a characteristic of pleomorphic xanthoastrocytoma and ganglioglioma and is an important prognosis factor also [51]. The genetic profile of these mutations indicates the fundamental possibility of their detection using $\mathrm{THz}$ technology that may have a direct impact not only on the verification of tumor histogenesis, but also on further tactics of surgical treatment.

However, numerous research efforts are required in order to make $\mathrm{THz}$ molecular diagnosis possible. Indeed, strong impact of free and bound water on the $\mathrm{THz}$ dielectric response of tissues should be suppressed, thus, unmasking spectral fingerprints of other endogenous molecular markers of a tumor.

\subsection{Multimodal diagnosis of brain tumors}

In general, the variability and heterogeneity of the resulting $\mathrm{THz}$ spectroscopy and imaging data somewhat reduce the sensitivity and specificity of these novel tools of intraoperative neurodiagnosis [18]. In order to increase the efficiency of $\mathrm{THz}$ technology in diagnosis of brain tumors, multimodal approaches may be applied by combining several modalities of tissue spectroscopy and imaging. In particular, it was shown in Ref. [43] that a favorable combination of preoperative Magnetic Resonance Imaging (MRI), White-Light Imaging (WLI), Green Fluorescence Protein (GFP) imaging, H\&E-stained histology, Optical Coherence Tomography (OCT), THz imaging and 5-ALA-induced fluorescence navigation allows to boost the performance of resultant system and to improve the tissue differentiation; see Fig. 6.

It is worth noting that pathogenetic links that determine the features of the distribution of water in the tumor tissue and, as a result, the characteristic features in the $\mathrm{THz}$ image, can arise not only in the framework of the oncological process. One of the common variants of brain pathology, accompanied by similar pathogenetic changes, is brain injury. On the one hand, this creates opportunities for the diagnosis of traumatic changes in brain tissue during surgery, which has been 
(a) MRI

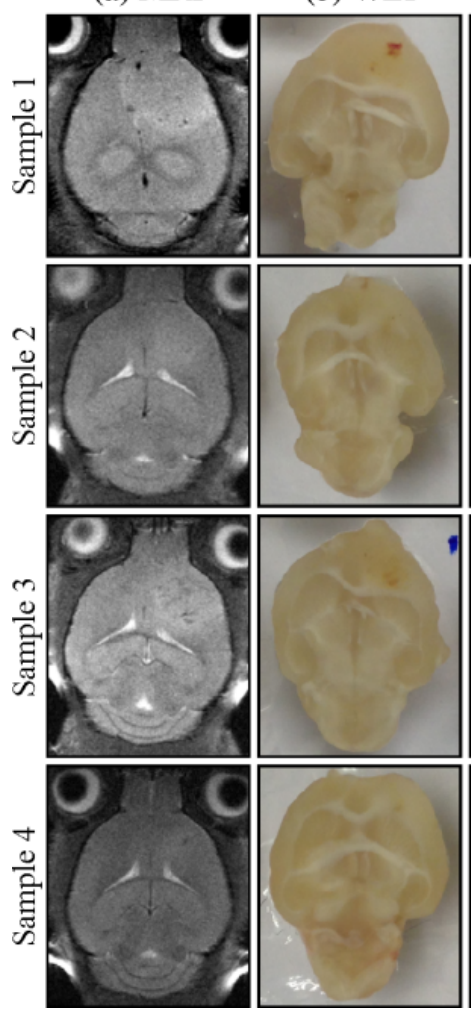

(c) GFPI
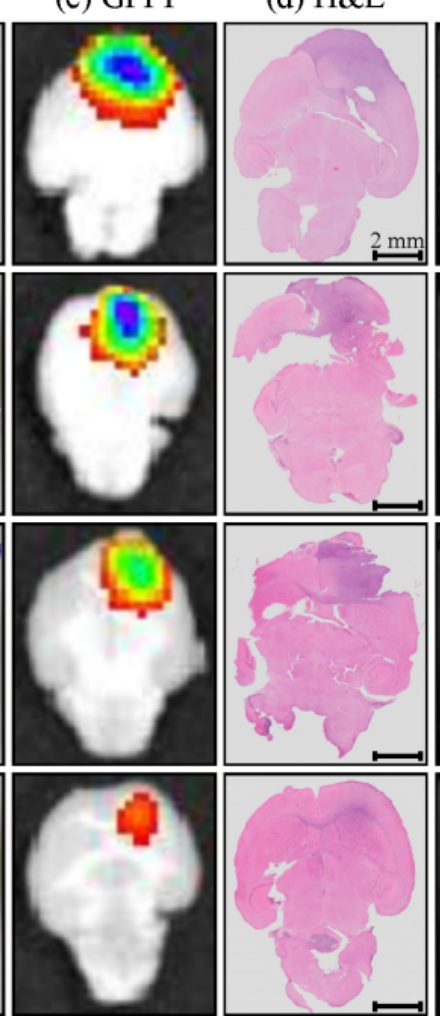

(d) $\mathrm{H} \& \mathrm{E}$

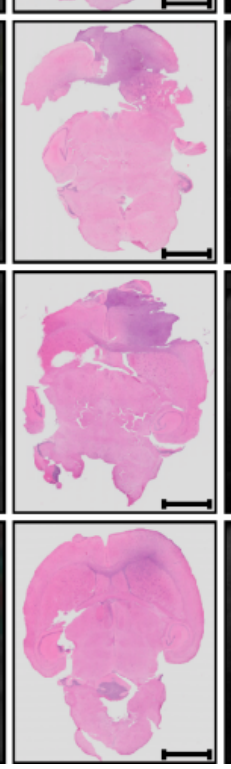

(e) OCT

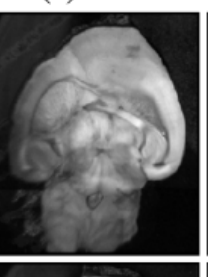

(f) THz-RI
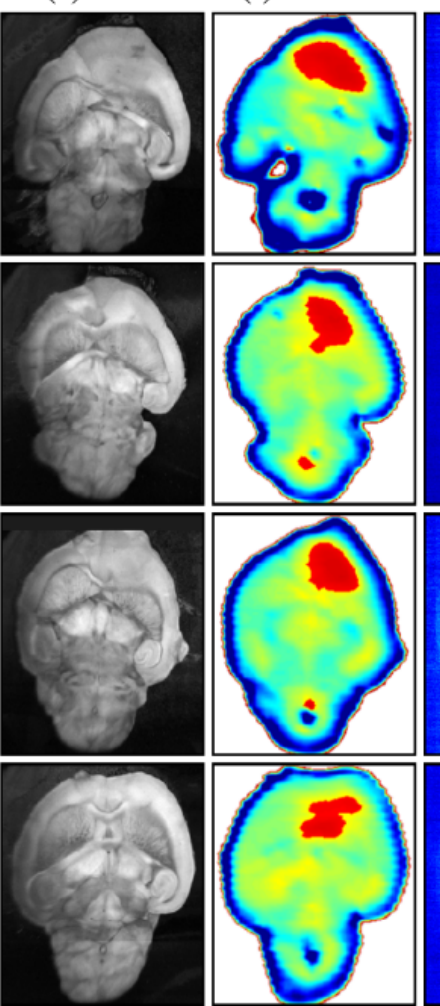

(g) ppIX
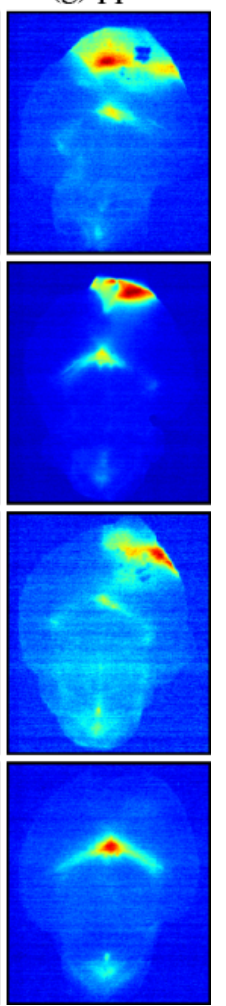

Fig. 6 Various imaging modalities of tissue imaging applied for differentiation between intact tissue and glioma models from 4 samples of whole mouse brain: (a) preoperative Magnetic Resonance Imaging (MRI); (b) White Light Imaging (WLI); (c) Green-Fluorescent Protein (GFP) imaging; (d) Hematoxylin and Eosin (H\&E)-stained histology; (e) Optical Coherence Tomography (OCT); (f) THz Reflection-mode Imaging (THz-RI); (g) 5-ALA-induced protoporphyrin IX fluorescence (ppIX) imaging. Adapted from Ref. [43], published by Springer Nature under a Creative Commons (CC BY) license.

proven in Ref. [52]. However, on the other hand, this intersection of pathogenetic links between traumatic injury and brain tumors makes it difficult to verify the pathological process, this difficulty can also be overcome using a multimodal approach for the pathology assessment.

\section{Conclusions}

In this review, fundamental possibility of using $\mathrm{THz}$ technology for the intraoperative diagnosis of CNS neoplasms was considered. Some problems that stand in the way of the implementation of the discussed technique in clinical practice are discussed. Most of these problems are fundamental and require continuation to clinical and paraclinical trials of $\mathrm{THz}$ technology in neurooncology. At the same time, advantages of $\mathrm{THz}$ diagnostics predetermine the prospect of further work on the translation of available fundamental developments in practice. Further progress in this area and deeper penetration of $\mathrm{THz}$ technologies into practice will be accompanied by the emergence of new problematic issues directly related to the clinical and medical aspects of the new diagnostic approach, including the ergonomics of $\mathrm{THz}$ equipment for surgery rooms, its biological safety and financial viability. The solution of all the questions posed at different stages of the $\mathrm{THz}$ diagnostic development in medicine requires further extensive research and engineering efforts.

\section{Disclosures}

The authors declare that there are no conflicts of interest related to this article.

\section{Acknowledgments}

The work was supported by the Russian Science Foundation (RSF), Project \# 18-12-00328. 


\section{References}

1. Q. Ostrom, H. Gittleman, G. Truitt, A. Boscia, C. Kruchko, and J. Barnholtz-Sloan, "CBTRUS statistical report: Primary brain and other central nervous system tumors diagnosed in the United States in 2011-2015," NeuroOncology 20(Supp14), iv1-iv86 (2018).

2. E. F. Chang, A. Clark, R. L. Jensen, M. Bernstein, A. Guha, G. Carrabba, D. Mukhopadhyay, W. Kim, L. M. Liau, S. M. Chang, J. S. Smith, M. S. Berger, and M. W. McDermott, "Multiinstitutional validation of the University of California at San Francisco low-grade glioma prognostic scoring system,” Journal of Neurosurgery 111(2), 203210 (2009).

3. M. Hefti, H. Mehdorn, I. Albert, and L. Dorner, "Fluorescence-guided surgery for malignant glioma: a review on aminolevulinic acid induced protoporphyrin ix photodynamic diagnostic in brain tumors," Current Medical Imaging Reviews 6(4), 254-258 (2010).

4. F. N. Obeidat, H. A. Awad, A. T. Mansour, M. H. Hajeer, M. A. Al-Jalabi, and L. E. Abudalu, "Accuracy of Frozen-Section Diagnosis of Brain Tumors: An 11-Year Experience from a Tertiary Care Center," Turkish Neurosurgery 29(2), 242-246 (2019).

5. W. Stummer, U. Pichlmeier, T. Meinel, O. D. Wiestler, F. Zanella, and H.-J. Reulen, "Fluorescence-guided surgery with 5-aminolevulinic acid for resection of malignant glioma: a randomised controlled multicentre phase III trial," The Lancet Oncology 7(5), 392-401 (2006).

6. M. Jermyn, J. Desroches, J. Mercier, K. St-Arnaud, M.-C. Guiot, F. Leblond, and K. Petrecca, "Raman spectroscopy detects distant invasive brain cancer cells centimeters beyond MRI capability in humans," Biomedical Optics Express 7(12), 5129-5137 (2016).

7. B. Liang, W. Liu, Q. Zhan, M. Li, M. Zhuang, Q. Huo, and L. J. Yao, "Impacts of the murine skull on highfrequency transcranial photoacoustic brain imaging," Journal of Biophotonics 12(7), e201800466 (2019).

8. F. Vase, N. MacKinnon, D. Farkas, and B. Kateb, "Review of the potential of optical technologies for cancer diagnosis in neurosurgery: a step toward intraoperative neurophotonics," Neurophotonics 4(1), 011010 (2016).

9. I. Dolganova, P. Aleksandrova, S.-I. Beshplav, N. Chernomyrdin, E. Dubyanskaya, S. Goryaynov, V. Kurlov, I. Reshetov, A. Potapov, V. Tuchin, and K. Zaytsev, "Wavelet-domain de-noising of OCT images of human brain malignant glioma," Proceedings of SPIE 10717, 107171X (2018).

10. Y.-S. Lee, Principles of Terahertz Science and Technology, Springer, 340 (2009).

11. K. Zaytsev, N. Chernomyrdin, K. Kudrin, I. Reshetov, and S. Yurchenko, "Terahertz spectroscopy of pigmentary skin nevi in vivo," Optics and Spectroscopy 119(3), 404-410 (2015).

12. Y. Sim, J. Park, K.-M. Ahn, C. Park, and J.-H. Son, "Terahertz imaging of excised oral cancer at frozen temperature," Biomedical Optics Express 4(8), 1413-1421 (2013).

13. C. Reid, A. Fitzgerald, G. Reese, R. Goldin, P. Tekkis, P. O'Kelly, E. Pickwell-MacPherson, A. Gibson, and V. Wallace, "Terahertz pulsed imaging of freshly excised human colonic tissues," Physics in Medicine \& Biology 56(14), 4333-4353 (2011).

14. A. Fitzgerald, V. Wallace, S. Pinder, A. Purushotham, P. O'Kelly, and P. Ashworth, "Classiffication of terahertzpulsed imaging data from excised breast tissue," Journal of Biomedical Optics 17(1), 016005 (2012).

15. Y. Ji, C. Park, H. Kim, S.-H. Kim, G. Lee, S. Noh, T.-I. Jeon, J.-H. Son, Y.-M. Huh, S. Haam, S. Oh, S. Lee, and J.-S. Suh, "Feasibility of terahertz reflectometry for discrimination of human early gastric cancers," Biomedical Optics Express 6(4), 1398-1406 (2015).

16. H. Chen, S.-H. Ma, W.-X. Yan, X.-M. Wu, and X.-Z. Wang, "The diagnosis of human liver cancer by using THz fiber-scanning near-field imaging," Chinese Physics Letters 30(3), 030702 (2013).

17. A. Gavdush, N. Chernomyrdin, K. Malakhov, S.-I. Beshplav, I. Dolganova, A. Kosyrkova, P. Nikitin, G. Musina, G. Katyba, I. Reshetov, O. Cherkasova, G. Komandin, V. Karasik, A. Potapov, V. Tuchin, and K. Zaytsev, "Terahertz spectroscopy of gelatin-embedded human brain gliomas of different grades: a road toward intraoperative THz diagnosis," Journal of Biomedical Optics 24(2), 027001 (2019).

18. O. A. Smolyanskaya, N. V. Chernomyrdin, A. A. Konovko, K. I. Zaytsev, I. A. Ozheredov, O. P. Cherkasova, M. M. Nazarov, J.-P. Guillet, S. A. Kozlov, Yu. V. Kistenev, J.-L. Coutaz, P. Mounaix, V. L. Vaks, J.-H. Son, H. Cheon, V. P. Wallace, Yu. Feldman, I. Popov, and V. V. Tuchin, "Terahertz biophotonics as a tool for studies of dielectric and spectral properties of biological tissues and liquids," Progress in Quantum Electronics 62, 1-77 (2018).

19. K. Cole, R. Cole, "Dispersion and absorption in dielectrics I. Alternating current characteristics," The Journal of Chemical Physics 9(4), 341-351 (1941).

20. K. Cole, R. Cole, "Dispersion and absorption in dielectrics II. Direct current characteristics," The Journal of Chemical Physics 10(2), 98-105 (1942).

21. D. Davidson, "Dielectric relaxation in liquids: I. The representation of relaxation behavior," Canadian Journal of Chemistry 39(3), 571-594 (1961).

22. S. Havriliak, S. Negami, “A complex plane analysis of dispersions in some polymer systems," Journal of Polymer Science Part C: Polymer Symposia 14(1), 99-117 (1966). 
23. E. Pickwell, B. E. Cole, A. J. Fitzgerald, V. P. Wallace, and M. Pepper, "Simulation of terahertz pulse propagation in biological systems," Applied Physics Letters 84(12), 2190-2192 (2004).

24. E. Pickwell, A. J. Fitzgerald, B. E. Cole, P. F. Taday, R. J. Pye, T. Ha, M. Pepper, and V. P. Wallace, "Simulating the response of terahertz radiation to basal cell carcinoma using ex vivo spectroscopy measurements," Journal of Biomedical Optics 10(6), 064021 (2005).

25. G. C. Walker, E. Berry, S. W. Smye, N. N. Zinov'ev, A. J. Fitzgerald, R. E. Miles, M. Chamberlain, and M. A. Smith, "Modelling the propagation of terahertz radiation through a tissue simulating phantom," Physics in Medicine \& Biology 49(10), 1853-1864 (2004).

26. A. J. Fitzgerald, E. Pickwell-MacPherson, and V. P. Wallace, "Use of finite difference time domain simulations and Debye theory for modelling the terahertz reflection response of normal and tumour breast tissue," PLOS ONE 9, e99291 (2014).

27. B. C. Q. Truong, H. D. Tuan, V. P. Wallace, A. J. Fitzgerald, and H. T. Nguyen, "The potential of the double Debye parameters to discriminate between basal cell carcinoma and normal skin," IEEE Transactions on Terahertz Science \& Technology 5(6), 990-998 (2015).

28. E. P. Parrott, S. M. Y. Sy, T. Blu, V. P. Wallace, and E. Pickwell-MacPherson, "Terahertz pulsed imaging in vivo: measurements and processing methods," Journal of Biomedical Optics 16(10), 106010 (2011).

29. S. J. Oh, S.-H. Kim, Y. B. Ji, K. Jeong, Y. Park, J. Yang, D. W. Park, S. K. Noh, S.-G. Kang, Y.-M. Huh, J.-H. Son, and J.-S. Suh, "Study of freshly excised brain tissues using terahertz imaging," Biomedical Optics Express 5(8), 2837-2842 (2014).

30. C. S. Joseph, A. N. Yaroslavsky, M. Al-Arashi, T. M. Goyette, J. C. Dickinson, A. J. Gatesman, B. W. Soper, C. M. Forgione, T. M. Horgan, E. J. Ehasz, R. H. Giles, and W. E. Nixon, "Terahertz spectroscopy of intrinsic biomarkers for non-melanoma skin cancer," Proceedings of SPIE 7215, 72150I (2009).

31. M. Ney, I. Abdulhalim, "Comprehensive Monte-Carlo simulator for optimization of imaging parameters for high sensitivity detection of skin cancer at the THz," Proceedings of SPIE 9721, 97210W (2016).

32. N. V. Chernomyrdin, A. S. Kucheryavenko, E. N. Rimskaya, I. N. Dolganova, V. A. Zhelnov, P. A. Karalkin, A. A. Gryadunova, I. V. Reshetov, D. V. Lavrukhin, D. S. Ponomarev, V. E. Karasik, and K. I. Zaytsev, "Terahertz microscope based on solid immersion effect for imaging of biological tissues," Optics \& Spectroscopy 126(5), 560-567 (2019).

33. A. Ishimaru, Electromagnetic wave propagation, radiation, and scattering: From fundamentals to applications, Wiley-IEEE Press, Piscataway, NJ, USA (2017).

34. P. Doradla, K. Alavi, C. Joseph, and R. Giles, "Detection of colon cancer by continuous-wave terahertz polarization imaging technique," Journal of Biomedical Optics 18(9), 090504 (2013).

35. V. Tuchin. Tissue optics: Light scattering methods and instruments for medical diagnostics, Third edition, SPIE Press, Bellingham, Washington, USA (2015).

36. K. I. Zaytsev, I. N. Dolganova, N. V. Chernomyrdin, G. M. Katyba, A. A. Gavdush, O. P. Cherkasova, G. A. Komandin, M. A. Shchedrina, A. N. Khodan, D. S. Ponomarev, I. V. Reshetov, V. E. Karasik, M. Skorobogatiy, V. N. Kurlov, and V. V. Tuchin, "The progress and perspectives of terahertz technology for diagnosis of neoplasms: A review," Journal of Optics 22(1), 013001 (2020).

37. G. M. Png, R. Flook, B.W.-H. Ng, and D. Abbott, "Terahertz spectroscopy of snap-frozen human brain tissue: an initial study," Electronics Letters 45(7), 343-345 (2009).

38. L. Shi, P. Shumyatsky, A. Rodríguez-Contreras, and R. Alfano, "Terahertz spectroscopy of brain tissue from a mouse model of Alzheimer's disease," Journal of Biomedical Optics 21(1), 015014 (2016).

39. K. Meng, T.-N. Chen, T. Chen, L.-G. Zhu, Q. Liu, Z. Li, F. Li, S.-C. Zhong, Z.-R. Li, H. Feng, and J.-H. Zhao, "Terahertz pulsed spectroscopy of paraffin-embedded brain glioma," Journal of Biomedical Optics 19(7), 077001 (2014).

40. S. Doblas, T. He, D. Saunders, J. Pearson, J. Hoyle, N. Smith, M. Lerner, and R. A. Towner, “Glioma morphology and tumor- induced vascular alterations revealed in seven rodent glioma models by in vivo magnetic resonance imaging and angiography," Journal of Magnetic Resonance Imaging 32(2), 267-275 (2010).

41. Y. Zou, J. Li, Y. Cui, P. Tang, L. Du, T. Chen, K. Meng, Q. Liu, H. Feng, J. Zhao, M. Chen, and L.-G. Zhu, "Terahertz spectroscopic diagnosis of myelin deficit brain in mice and rhesus monkey with chemometric techniques," Scientific Reports 7, 5176 (2017).

42. S. Yamaguchi, Y. Fukushi, O. Kubota, T. Itsuji, T. Ouchi, and S. Yamamoto, "Brain tumor imaging of rat fresh tissue using terahertz spectroscopy," Scientific Reports 6, 30124 (2016).

43. Y. B. Ji, S. J. Oh, S.-G. Kang, J. Heo, S.-H. Kim, Y. Choi, S. Song, H. Y. Son, S. H. Kim, J. H. Lee, S. J. Haam, Y. M. Huh, J. H. Chang, C. Joo, and J.-S. Suh, "Terahertz reflectometry imaging for low and high grade gliomas," Scientific Reports 6, 36040 (2016).

44. N. V. Chernomyrdin, A. A. Gavdush, S.-I. T. Beshplav, K. M. Malakhov, A. S. Kucheryavenko, G. M. Katyba, I. N. Dolganova, S. A. Goryaynov, V. E. Karasik, I. E. Spektor, V. N. Kurlov, S. O. Yurchenko, G. A. Komandin, A. A. Potapov, V. V. Tuchin, and K. I. Zaytsev, "In vitro terahertz spectroscopy of gelatin-embedded human brain tumors: a pilot study,” Proceedings of SPIE 10716, 107160S (2018). 
45. S. Fan, B. Ung, E. P. J. Parrott, and E. Pickwell-MacPherson, "Gelatin embedding: a novel way to preserve biological samples for terahertz imaging and spectroscopy," Physics in Medicine \& Biology 60(7), 2703 (2015).

46. B. M. Fischer, M. Walther, and P. U. Jepsen, "Far-infrared vibrational modes of DNA components studied by terahertz time-domain spectroscopy," Physics in Medicine \& Biology 47(21), 3807-3814 (2002).

47. E. Pickwell-MacPherson, V. P. Wallace, "Terahertz pulsed imaging - A potential medical imaging modality," Photodiagnosis and Photodynamic Therapy 6(2), 128-134 (2009).

48. M. Tang, M. Zhang, S. Yan, L. Xia, Z. Yang, C. Du, H. L. Cui, and D. Wei, "Detection of DNA oligonucleotides with base mutations by terahertz spectroscopy and microstructures," PLOS ONE 13(1), e0191515 (2018).

49. P. Wesseling, D. Capper, "WHO 2016 Classification of gliomas," Neuropathology and Applied Neurobiology 44(2), 139-150 (2018).

50. R. Chen, M. Smith-Cohn, A. L. Cohen, and H. Colman, "Glioma Subclassifications and Their Clinical Significance," Neurotherapeutics 14(2), 284-297 (2017).

51. X. Chen, C. Pan, P. Zhang, C. Xu, Y. Sun, H. Yu, Y. Wu, Y. Geng, P. Zuo, Z. Wu, J. Zhang, and L. Zhang, "BRAF V600E mutation is a significant prognosticator of the tumour regrowth rate in brainstem gangliogliomas," Journal of Clinical Neuroscience 46, 50-57 (2017).

52. H. Zhao, Y. Wang, L. Chen, J. Shi, K. Ma, L. Tang, D. Xu, J. Yao, H. Feng, and T. Chen, "High-sensitivity terahertz imaging of traumatic brain injury in a rat model," Journal of Biomedical Optics 23(3), 036015 (2018). 